\title{
Traffic Oscillations Mitigation in Vehicle Platoon Using a Car-Following Control Model for Connected and Autonomous Vehicle
}

\author{
Zhijun Gao $\mathbb{D},{ }^{1}$ Jiangfeng Wang $\mathbb{D},{ }^{1}$ Xi Zhang, ${ }^{1}$ Jiakuan Dong, ${ }^{1}$ Lei Chen, ${ }^{1}$ Xuedong Yan $\mathbb{D}^{1},{ }^{1}$ \\ and Wei Zhang ${ }^{2}$ \\ ${ }^{1}$ Key Laboratory of Transport Industry of Big Data Application Technologies for Comprehensive Transport, Ministry of Transport, \\ Beijing Jiaotong University, Beijing 100044, China \\ ${ }^{2}$ China Academy of Transportation Sciences, Ministry of Communications, Beijing 100029, China
}

Correspondence should be addressed to Jiangfeng Wang; wangjiangfeng@bjtu.edu.cn

Received 30 April 2019; Revised 22 July 2019; Accepted 21 August 2019; Published 3 December 2019

Guest Editor: Hao Yu

Copyright @ 2019 Zhijun Gao et al. This is an open access article distributed under the Creative Commons Attribution License, which permits unrestricted use, distribution, and reproduction in any medium, provided the original work is properly cited.

\begin{abstract}
Traffic oscillations often occur in road traffic, they make traffic flow unstable, unsafe and inefficient. Emerging connected and autonomous vehicle (CAV) technologies are potential solutions to mitigating the traffic oscillations for the advantages that CAVs are controllable and cooperative. In order to study a control strategy and the effectiveness of CAVs in mitigating traffic oscillations and improving traffic flow and analyse the characteristics of homogeneous traffic flow made up of CAVs and heterogeneous traffic flow made up of CAVs and RVs when traffic oscillations appear in traffic flow. Firstly, the formation and propagation of traffic oscillations in a platoon of RVs are simulated and analysed. Then, a car-following control model is built to control the longitudinal motion of CAVs, and real-time information of preceding CAV is used in the model and this can make the motion of CAVs more cooperative. The model reflects an idea named "slow-in" and "fast-out," and this idea is helpful to mitigate traffic oscillations. Then, numerical simulations of homogeneous traffic flow of a platoon of CAVs and simulations of heterogeneous traffic flow containing CAVs and RVs are conducted, and different penetration rates $(0,0.2,0.4,0.6,0.8$, and 1$)$ of CAVs are considered in the simulations of heterogeneous traffic flow. The characteristics and evolution of traffic flow are analysed and some indexes reflecting traffic efficiency and stability are calculated and analysed. Simulation results show that there are smaller velocity fluctuation, less stopping time and shorter length of road occupied when vehicle platoon contains CAVs (penetration rates are from 0.2 to 1) compared to the platoon containing only RVs (without CAVs). As for the heterogeneous traffic flow containing CAVs and RVs, these three indexes decrease with the increase of penetration rates (from 0.2 to 1 ) of CAVs. These results indicate that CAVs with the car-following control model in vehicle platoon are beneficial for mitigating traffic oscillations and improving traffic flow.
\end{abstract}

\section{Introduction}

Traffic oscillations often happen in road traffic. When vehicles are captured by traffic oscillations, they have to decelerate one by one and move slowly or even stop. Traffic oscillations have extremely negative effects on traffic flow, e.g., high risk of accidents, high fuel consumptions, and emissions and low traffic efficiency. Previous studies [1] found that a slight fluctuation can destroy the initial homogeneous motion and stop-and-go waves gradually form. Many studies [2-5] used NGSIM dataset [6] to explore the characteristics of traffic oscillations and stop-and-go waves. Laval and Leclercq [2] and Laval et al. [3] studied the formation and propagation of traffic oscillations and Chen et al. $[4,5]$ analysed the impacts of driver characteristics on traffic oscillations. Considering the negative impact of oscillations on traffic flow, many studies provided jam-absorption driving strategies to mitigate traffic oscillations. Nishi et al. [7] designed a theoretical framework of driving strategy for a single vehicle based on a basic idea called "slow-in" and "fast-out," that is when a vehicle is approaching traffic oscillations it moves slowly then it moves fast after getting rid of the oscillations. The driving strategy in $\mathrm{He}$ et al. [8] has a similar idea. Taniguchi et al. [9] proposed a car-following model to absorb traffic oscillations and avoid secondary traffic jam in 
vehicle platoon. Caruntu et al. [10] designed vehicle controllers for mitigating traffic oscillations based on ensuring the string stability of vehicle platoon.

Nowadays, connected vehicle (CV) technologies are thought as an effective approach to ensure safer and more efficient traffic. CV technologies make it possible for vehicles to exchange real-time information mutually. Many researches found that connected vehicles (CVs) have positive impacts on traffic flow [11-13]. In CV environment, in-vehicle devices can receive some messages about surrounding traffic from surrounding vehicles or road infrastructures, these messages can assist drivers to drive safely and efficiently compared with conventional traffic. Some field experiments $[14,15]$ indicated that drivers have shorter reaction time in $\mathrm{CV}$ environment. $\mathrm{CV}$ technologies can provide drivers much information about surrounding traffic, which have positive impact on car-following behaviour. Many researchers attempt to model car-following behaviour in $\mathrm{CV}$ environment and compare the differences between behaviours in $\mathrm{CV}$ environment and conventional traffic. Based on the traditional car-following models, several extended car following models for CV environment were proposed to analyse the impacts of vehicle-to-vehicle communication $[16,17]$, connected cruise control strategy [18], speed guidance at signal intersection [19] and communication delay [20] on traffic flow. At the same time, based on the analysis of traffic oscillations, CV technologies and car-following behaviour, more and more studies were conducted to suppress oscillations and smooth traffic flow considering $\mathrm{CV}$ technologies. To alleviate impact of traffic oscillations in the vehicle platoon, Kamal et al. [21] designed a predictive control strategy for a vehicle in a platoon of vehicles using the real-time information of preceding vehicles. Some variable speed limits strategies in CV environment are designed to eliminate stop-and-go waves [12] and improve traffic flow [22].

On the other hand, with the development of autonomous vehicle (AV) technologies, AVs run on real roads and participate in conventional traffic is no longer a utopia. In fact, AV is just a robot controlled by computer programming, it means that $\mathrm{AV}$ is controllable and has few uncertainties relative to human drivers. The good characteristics of AVs will make the traffic management and organization transform from infrastructure-based methods to vehicle-based methods. Instead of using variable speed limits strategies, many studies focused on controlling the motion of one or several AVs to mitigate traffic waves and smooth traffic flow. Field experiments conducted by Stern et al. [23] indicated that a simple control strategy for only one AV could dampen traffic waves in a circular track. However, on-board sensors of AV may have blind area of perception and this disadvantage prevents AV from moving safely and efficiently. Adding CV technologies into AV can make AV has over-the-horizon perceptions, shares real-time information with other AVs and overcomes the disadvantage, and this is the concept of CAVs. The combination of CV technologies and AV technologies will make the vehicle-based traffic control methods more cooperative and effective. Some control strategies $[24,25]$ use several CAVs or one CAV and several CVs to mitigate traffic oscillations in traffic flow. Ge et al. [25] designed a longitudinal controller for a CAV, it can receive real-time motion information from one or more preceding CVs driven by human, and field experiments were conducted and the results shown that the control strategy of CAV was helpful to mitigate traffic waves and improve safety and energy efficiency.

In order to use CAVs to mitigate traffic oscillations, the key point is how to control the longitudinal motion of CAVs when oscillations appear and propagate in vehicle platoon. Considering the effectiveness of the idea of "slow-in" and "fastout" [7] in mitigating traffic oscillations and considering the technologies that CAVs can share real-time state information with each other, we propose a car-following control model for CAVs to make CAVs move cooperatively and to mitigate traffic oscillations based on this idea as well as this technologies in this paper. In order to study the ability of mitigating traffic oscillations of the proposed car-following control model of CAVs, the proposed model will be verified in heterogeneous traffic flow made up of CAVs and regular vehicles (RVs), and the CAVs and RVs distribute randomly in vehicle platoon. At the same time, considering the limitation of using field tests to study the effectiveness of CAVs in mitigating traffic oscillations in large-scale traffic flow, this study will use simulation methods to overcome this limitation. In addition, considering that the motion characteristic of CAV should have some similarity with RV, we want to select an existing car-following model and build our car-following control model for CAV based on the existing model. In classical traffic flow theory, the car-following behaviour is modelled using the response-stimulus mechanism which can be described as driver has a response (velocity or acceleration changing) to stimulus (velocity difference or space headway) from preceding vehicle [26-28]. Bando et al. [29] developed a car-following model named optimal velocity (OV) model, this model uses the difference between the actual velocity and driver expected velocity which is decided by the space headway to adjust vehicle's acceleration. OV model is a mature and wideused model in simulating car-following behaviour. Many different car-following models [30-36] extended from OV model were developed to better describe car-following behaviour with higher precision or to make model applicable to various situation. So, in this paper, we develop the car-following control strategy for CAV based on OV model.

The remainder of this paper is organized as follows: Section 2 describes the OV model and analyses the formation and propagation of traffic oscillations in a vehicle platoon. A car-following control model based on OV model is proposed for the longitudinal motion of CAV in Section 3, and the model uses some information of the preceding vehicle and reflects the idea of "slow-in" and "fast-out." In Section 4, numerical simulations with homogeneous traffic flow containing only CAVs and with heterogeneous traffic flow containing CAVs and RVs are conducted, and the effectiveness that CAVs mitigate traffic oscillations are analysed. Finally, a conclusion is made.

\section{Formation and Propagation of Traffic Oscillations on a Single Lane}

Car-following behaviour is a general phenomenon in traffic flow, which has great negative effects on mobility and safety. 
When some consecutive vehicles form a platoon and travel at an equal velocity and constant space headway, traffic efficiency is high. On the contrary, once a vehicle's velocity has a sudden drop due to a perturbation, traffic flow will be made unstable and traffic oscillations will appear in platoon. As a result, there is a lower road capacity and a higher crash risk. OV model performs well in simulating traffic oscillations and congestion. Consequently OV model is applied to simulate the formation and propagation of traffic oscillations on a single lane. In OV model, driver uses the difference between vehicle's actual velocity and his expected velocity to adjust vehicle's acceleration. OV model [29] can be formulated as following:

$$
\frac{d v_{n+1}}{d t}(t)=\alpha\left\{V\left[\Delta x_{n+1}(t)\right]-v_{n+1}(t)\right\},
$$

where $n$ is the index number of vehicle, $\left(d v_{n+1} / d t\right)(t)$, $V\left[\Delta x_{n+1}(t)\right]$ and $v_{n+1}(t)$ are acceleration, optimal velocity (defined by Equation (2)) and velocity of vehicle $(n+1)$ at time $t$, respectively. $\alpha$ is driver's sensitivity parameter which is the reciprocal of the delay time that is required to reach the optimal velocity. Optimal velocity function developed by Helbing and Tilch [30] is adopted in this paper and it can be formulated as below

$$
V\left[\Delta x_{n+1}(t)\right]=V_{1}+V_{2} \tanh \left\{C_{1}\left[\Delta x_{n+1}(t)-l_{n+1}\right]+C_{2}\right\},(2)
$$

where $\Delta x_{n+1}(t)$ and $l_{n+1}$ are the space headway at time $t$ and minimum safe headway between vehicle $n+1$ and its preceding vehicle $n$. The constants are calibrated [30] as $\alpha=0.85 \mathrm{~s}^{-1}$, $V_{1}=6.75 \mathrm{~m} / \mathrm{s}, V_{2}=7.91 \mathrm{~m} / \mathrm{s}, C_{1}=0.13 \mathrm{~m}^{-1}$, and $C_{2}=1.57$.

As Equations (1) and (2) mentioned above, the optimal velocity is a function of space headway. When the actual velocity is equal to optimal velocity, acceleration is 0 and space headway and velocity will be constants.

To illustrate traffic oscillations in a platoon using OV model, a scenario is presumed that a vehicle platoon consisting of 100 vehicles is traveling on a straight lane and the leading vehicle of the platoon decelerates suddenly and then accelerates. In our scenario, initial space headway between every two consecutive vehicles is $\Delta x_{n+1}=26.75 \mathrm{~m}$, initial velocity of all vehicles in platoon is $V(26.75)=13.47 \mathrm{~m} / \mathrm{s}$, and the vehicle length is $l_{n+1}=5$. The maximum velocity of vehicle is $13.47 \mathrm{~m} / \mathrm{s}$, the maximum deceleration is comfortable deceleration which is $-3 \mathrm{~m} / \mathrm{s}^{2}$ and the maximum acceleration is comfortable acceleration which is $3 \mathrm{~m} / \mathrm{s}^{2}$.

The total simulation time is $300 \mathrm{~s}$ and time step of simulation is $0.1 \mathrm{~s}$. Simulation starts from $t=0$ sand vehicle platoon moves stably at the initial velocity for $50 \mathrm{~s}$. The leading vehicle decelerates from $t=51 \mathrm{~s}$ to $t=53 \mathrm{~s}$ with an deceleration of $-3 \mathrm{~m} / \mathrm{s}^{2}$ and drives at a uniform velocity from $t=54 \mathrm{~s}$ to $t=55 \mathrm{~s}$ and accelerates from $t=56 \mathrm{~s}$ to $t=58 \mathrm{~s}$ with an acceleration of $3 \mathrm{~m} / \mathrm{s}^{2}$ and drives at maximum velocity from $t=59 \mathrm{~s}$ to $t=300 \mathrm{~s}$. Traffic oscillations occur in the platoon because of the velocity fluctuation of the leading vehicle, and the analysis about the traffic oscillations is conducted as following.

All 100 vehicles are sequentially numbered with $1,2 \ldots$ and 100 respectively as their ID and trajectories are marked with $a$ " $v$ " and the ID number for corresponding vehicle in figures. Figure 1 shows time-space trajectories of 11 samples of vehicles. Within the first $50 \mathrm{~s}$, every two consecutive vehicles have

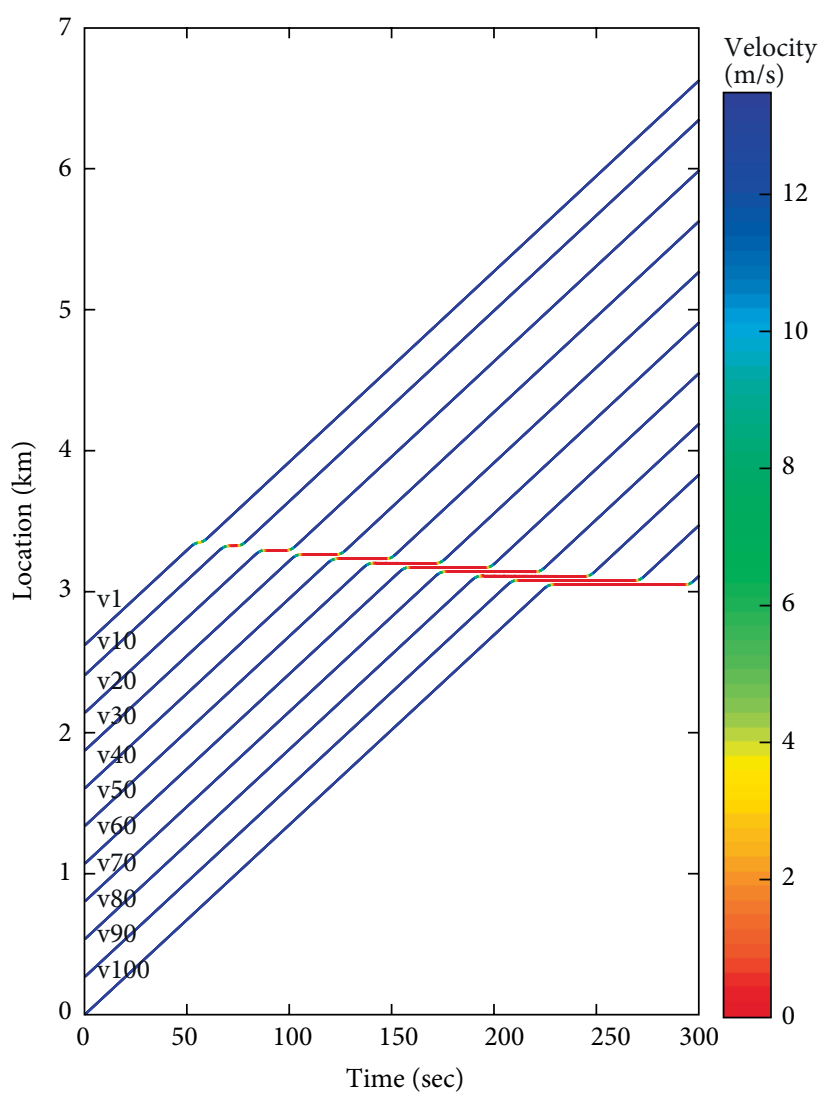

FIgURE 1: Time-space trajectories of 11 samples of vehicles.

equal and constant space headway and the vehicle platoon moves ahead stably. Traffic oscillations form at $t=51 \mathrm{~s}$ and propagate upstream gradually and make the platoon unstable locally. As the oscillations propagate, upstream vehicles are captured for longer time and move at low velocity or even stop. After being captured by the traffic oscillations for a period of time, vehicles will get rid of the oscillations and accelerate gradually and move at maximum velocity finally. The last vehicle $\left(100^{\text {th }}\right.$ vehicle) begins to move at $t=293 \mathrm{~s}$ after stopping for $63 \mathrm{~s}$ and it accelerates quickly to maximum velocity and a stable vehicle platoon finally forms again. It can also be noticed from Figure 1 that the space headway between vehicles in the final stable platoon is longer than that of original stable platoon, so the platoon occupies longer road and this phenomenon can also be reflected in Figure 2.

Figure 2 shows that in the first $50 \mathrm{~s}$, the spaces between $1^{\text {st }}$ vehicle and 99 following vehicles are constant. Length of road occupied by the vehicles platoon is $2.65 \mathrm{~km}$ and it can be calculated by space between $1^{\text {st }}$ vehicle and $100^{\text {th }}$ vehicle. Within $t=51-53 \mathrm{~s}, 1^{\text {st }}$ vehicle decelerates and spaces between $1^{\text {st }}$ vehicle and 99 following vehicles reduce and the minimum length of road occupied is $2.60 \mathrm{~km}$. After $t=54 \mathrm{~s}, 1^{\text {st }}$ vehicle begins to accelerate and reach the maximum velocity at $t=59 \mathrm{~s}$. However, after being captured by traffic oscillations sequentially, following vehicles have to decelerate more and more intensively and even stop for a period of time, and every vehicle has a larger space headway between itself and $1^{\text {st }}$ 


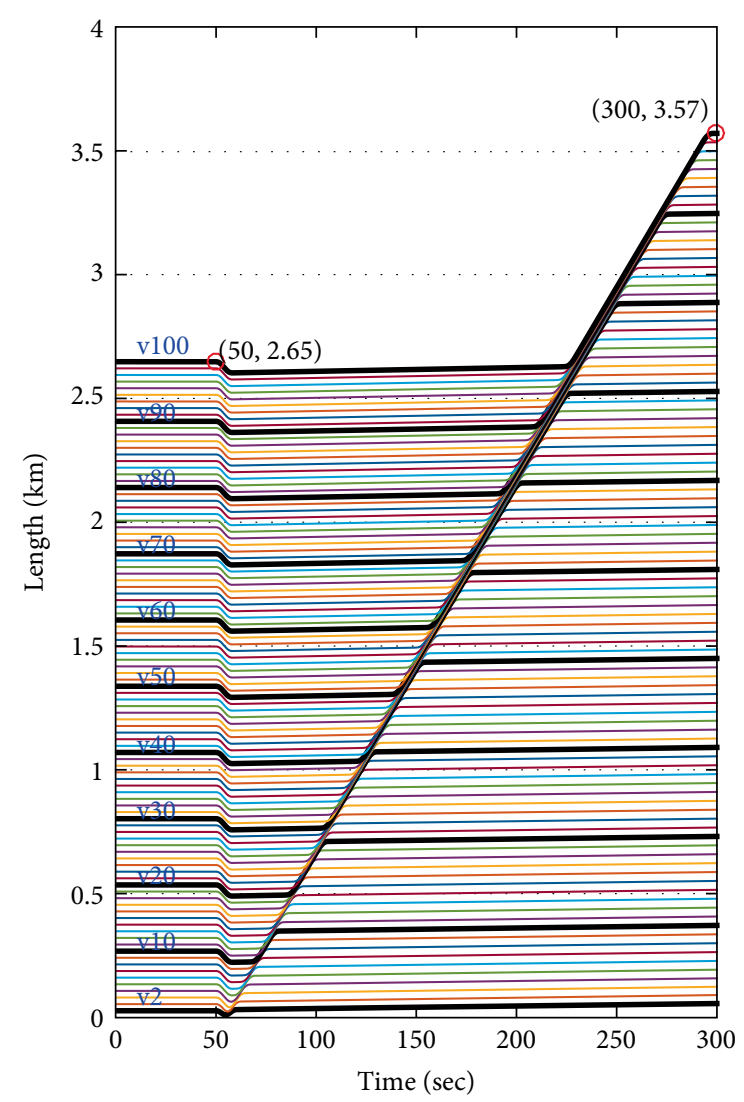

FIGURE 2: Space headway between 1st vehicle and 99 following vehicles.

vehicle after its' decelerating and even stopping. Finally, total length of road occupied is $3.57 \mathrm{~km}, 0.92 \mathrm{~km}$ longer than the first $50 \mathrm{~s}$.

Figure 3 illustrates fluctuation of vehicles' velocity. After decelerating for a short time, $1^{\text {st }}$ vehicle returns to its initial velocity, however, the traffic oscillations are caused and propagate upstream. With the increase of vehicle ID, vehicle's velocity fluctuation will be larger. As traffic oscillations propagate upstream, approximate $8^{\text {th }}$ vehicle has to stop for a short time and all the upstream vehicles behind $8^{\text {th }}$ vehicle have to stop for longer and longer time. This trend is easily reflected in Figure 4, and we can see the stopping time of vehicle behind $8^{\text {th }}$ vehicle increases gradually with the increase of ID number of vehicles.

The traffic oscillations phenomenon and analysis above motivate us to use emerging CAV technologies to improve traffic flow. We will explore how CAVs in homogeneous traffic flow and CAVs in mixed traffic flow (containing both CAVs and RVs) can mitigate traffic oscillations. In order to verify our idea, a car-following control model for CAV is necessary to be developed at next section.

\section{A Car-Following Control Model for CAV}

3.1. The Idea of "Slow-In" and "Fast-Out". Considering the advantage that CAVs is controllable, when traffic oscillations
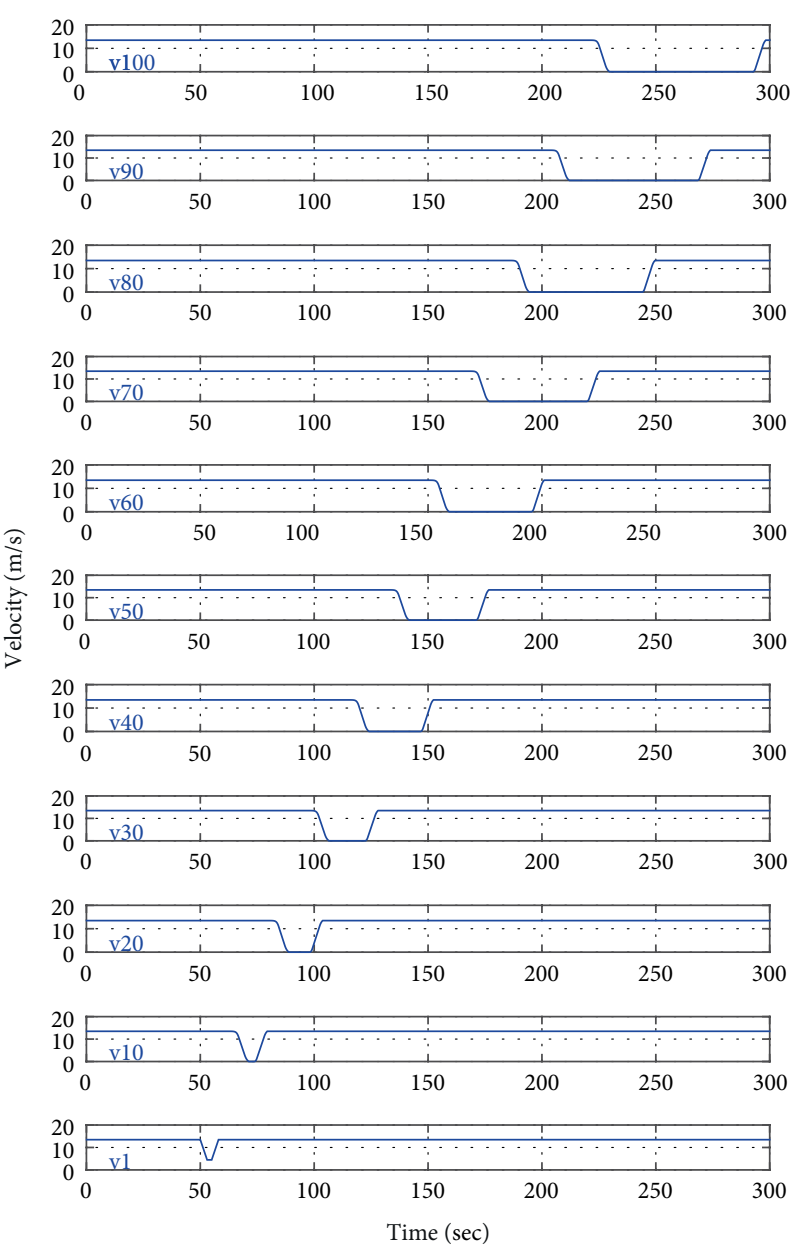

FIgURE 3: Velocity trajectories of 11 samples of vehicles.

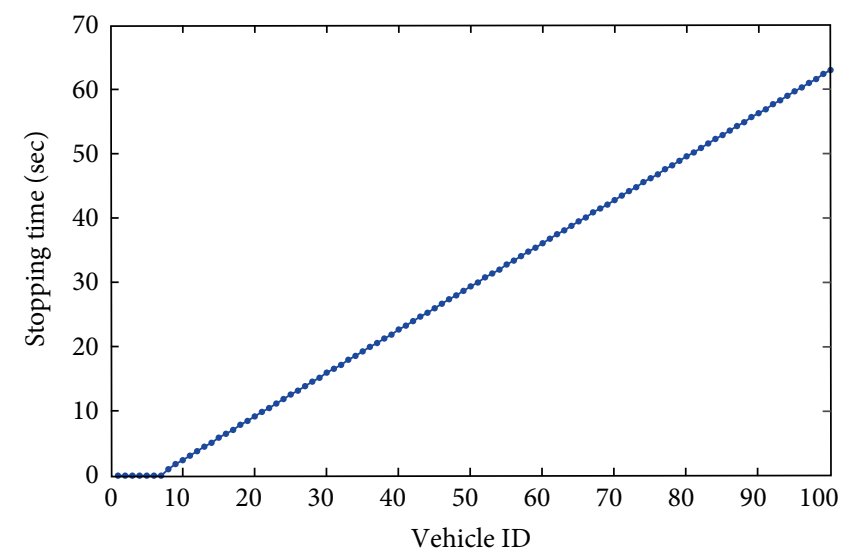

FIGURE 4: Stopping time of vehicles in upstream platoon.

appear in traffic flow, we can design motion strategy for CAVs to make CAVs move cooperatively, to alleviate the drop of velocity and to mitigate traffic oscillation. There is an effective idea named "slow-in" and "fast-out" [7], the meaning of this idea is that when vehicle is approaching traffic oscillations it moves slowly, and after getting rid of the oscillations, it moves fast. The oscillations can be mitigated if vehicles move 


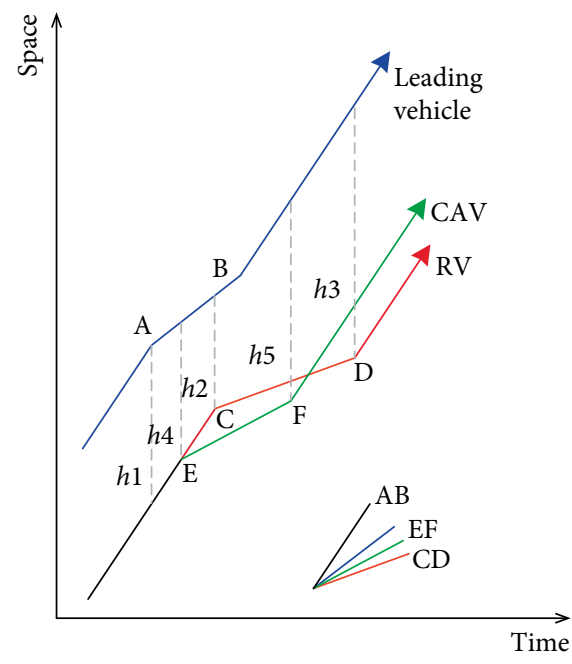

FIGURE 5: Illustration of the idea of "slow-in" and "fast-out."

according to this idea when oscillations occur in traffic flow. In addition, when a CAV follows another CAV, the following one can receive real-time information of the leading one. If the velocity of the leading CAV has a drop, the following CAV can realize very quickly with less reaction time (this reaction time is negligible) than human drivers. When the following CAV realizes the velocity drop of leading CAV, it moves according to the idea of "slow-in" and "fast-out," the traffic oscillation can be mitigated.

Figure 5 visualizes the idea of "slow-in" and "fast-out" by the time-space trajectories of a CAV and a RV in same carfollowing scenario. In Figure 5, blue line represents leading vehicle' time-space trajectories, red line and green one present trajectories of a vehicle which is a RV and a vehicle which is a $\mathrm{CAV}$, respectively. Black line is the same trajectories of CAV and $\mathrm{RV}$ at initial period. Blue line $\mathrm{AB}$ shows that the leading vehicle runs at a smaller velocity after it decelerates at point A. Two lines at both ends of line $A B$ represent that vehicle runs at maximum velocity, and red line $\mathrm{CD}$ and green line $\mathrm{EF}$ have similar meanings with blue line AB. Every grey vertical dashed line marked with an " $h$ " and a number represents space headway between the two vehicles at different time.

In traditional traffic, the following RV begins to decelerate at point $\mathrm{C}$ after driver's several seconds of reaction time during which vehicle is running in original maximum velocity. While a following CAV can decelerate earlier at point $\mathrm{E}$ after it receives the leading vehicle's real-time messages of velocity drop at point A. RV and CAV begin to accelerate and run at maximum velocity at points $\mathrm{D}$ and $\mathrm{F}$, respectively.

The four lines are gathered at the bottom right corner of the figure to compare the slopes of time-space trajectories lines as well as velocities, the bigger the slope means the greater the velocity. The black line without any mark represents vehicle's maximum velocity, which corresponds to the lines at both ends of lines $A B, C D$, and EF. Every line among three marked with two letters corresponds and parallels with line segment of trajectories marked with same two letters.
As for space headway at different time, $h 2<h 4$ $<h 1<h 5<h 3$ and it can be explained as below:

(i) $h 2<h 4$ : CAV decelerates earlier than RV.

(ii) $h 4<h 1$ : CAV decelerates later than leading vehicle.

(iii) $h 1<h 5$ : CAV travels longer time at lower velocity compared with leading vehicle.

(iv) $h 5<h 3$ : CAV accelerates earlier than RV.

After a same traffic oscillation, final space headway between following CAV and leading vehicle is less than that between following RV and leading vehicle, and this is benefit for saving road space. In addition, following CAV has longer space headway with leading vehicle than following RV in the circumstance of leading vehicle decelerating suddenly, which is a benefit for collision avoidance. Based on the above analysis, a car-following control model for CAV will be developed in next part.

3.2. Car-Following Control Model for CAV. In this part, a car-following control model for CAV is built based on the idea of "slow-in" and "fast-out." We introduce the real-time information of preceding vehicle into OV model to reflect this idea. The car-following control model for CAV can be formulated as follows:

$$
\frac{d v_{n+1}}{d t}(t)=\alpha\left\{V\left[\Delta x_{n+1}(t)\right]-v_{n+1}(t)\right\}+f(I)
$$

where $I$ refers to preceding vehicle's real-time information, $f(I)$ denotes how the information $I$ affects $\left(d v_{n+1} / d t\right)(t)$.

As a significant parameter reflecting vehicle's real-time motion state, acceleration of preceding vehicle is introduced into Equation (3) for the car-following control model for CAV:

$$
\frac{d v_{n+1}}{d t}(t)=\alpha\left\{V\left[\Delta x_{n+1}(t)\right]-v_{n+1}(t)\right\}+f\left[a_{n}(t), I^{\prime}\right],
$$

where $a_{n}(t)$ is preceding vehicle's deceleration, $I^{\prime}$ represents other information.

In general, the smaller space between two vehicles means more sensitive reflection of following vehicle to preceding vehicle. Therefore, vehicles' space headway should be contained in model and Equation (4) can be converted as followings:

$$
\frac{d v_{n+1}}{d t}(t)=\alpha\left\{V\left[\Delta x_{n+1}(t)\right]-v_{n+1}(t)\right\}+\frac{a_{n}(t)}{\Delta x_{n+1}} .
$$

As we can see from the Equation (5), when the preceding vehicle decelerates, the following CAV will move slower than the following RV. While, when the preceding vehicle accelerates, the following CAV will move quicker than the following $\mathrm{RV}$. In addition, this car-following control model is developed by introducing the preceding vehicle's Acceleration into OV model, which is named as AOV model. It should be noted that inter-vehicle communication delay is not considered in AOV 


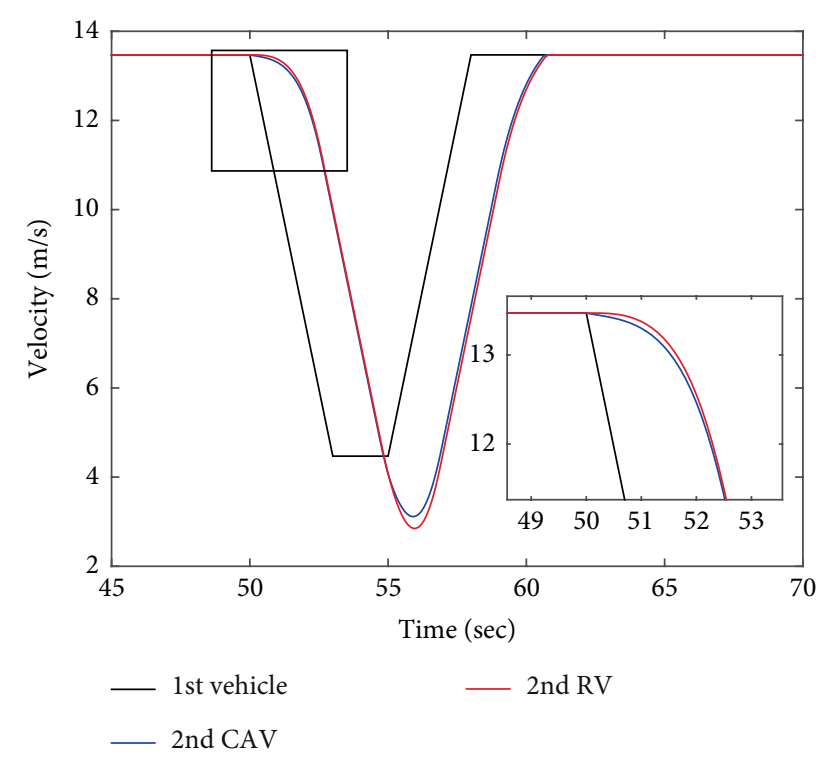

FIGURE 6: Velocity trajectories of 2nd vehicle and 1st vehicle in $45-70 \mathrm{~s}$ in two simulations.

model for the reason that there is negligible delay in advanced inter-vehicle communication technologies.

\section{Case Study}

In this section, we will verify whether the proposed car-following control model for CAV can mitigate traffic oscillations in homogeneous traffic flow containing only CAVs and in heterogeneous traffic flow containing CAVs and RVs.

\subsection{Numerical Simulation of Homogeneous Traffic Flow}

Containing Only CAVs. In this part, simulation is conducted to simulate the homogeneous traffic flow containing only CAVs when traffic oscillations appear in the traffic flow. All the 100 vehicles in simulation are CAVs and the leading vehicle of the platoon moves according to specified velocity and acceleration and remaining 99 CAVs move according to AOV model. The simulation scenario in this section is same as the simulation scenario in Section 2, that means the original velocity, space headway between vehicles, maximum acceleration/deceleration and perturbation caused by the leading vehicle of the platoon are same with simulation in Section 2. The analyses of simulation results are as following.

Velocity trajectories of the $2^{\text {nd }}$ vehicle and the $1^{\text {st }}$ vehicle in $45-70 \mathrm{~s}$ in two simulations are presented in Figure 6. Velocity trajectories of the $1^{\text {st }}$ vehicle in two simulations are same. At $t=51 \mathrm{~s}$, the $1^{\text {st }}$ vehicle brakes suddenly and decelerates to velocity of $4.47 \mathrm{~m} / \mathrm{s}$ after $3 \mathrm{~s}$ and then it keeps this velocity for $2 \mathrm{~s}$ and it accelerates and reaches velocity of $13.47 \mathrm{~m} / \mathrm{s}$ at $t=59$ s finally. When the $1^{\text {st }}$ vehicle decelerates, the $2^{\text {nd }}$ vehicle also decelerates to avoid collisions. The velocity fluctuation process of the $2^{\text {nd }}$ vehicle lasts about $11 \mathrm{~s}$ in two simulations, at the first $5 \mathrm{~s}$ of fluctuation, velocity of the $2^{\text {nd }} \mathrm{CAV}$ that moves according to AOV model is smaller than that of the $2^{\text {nd }} \mathrm{RV}$ that moves according to OV model, while at the last $6 \mathrm{~s}$ of
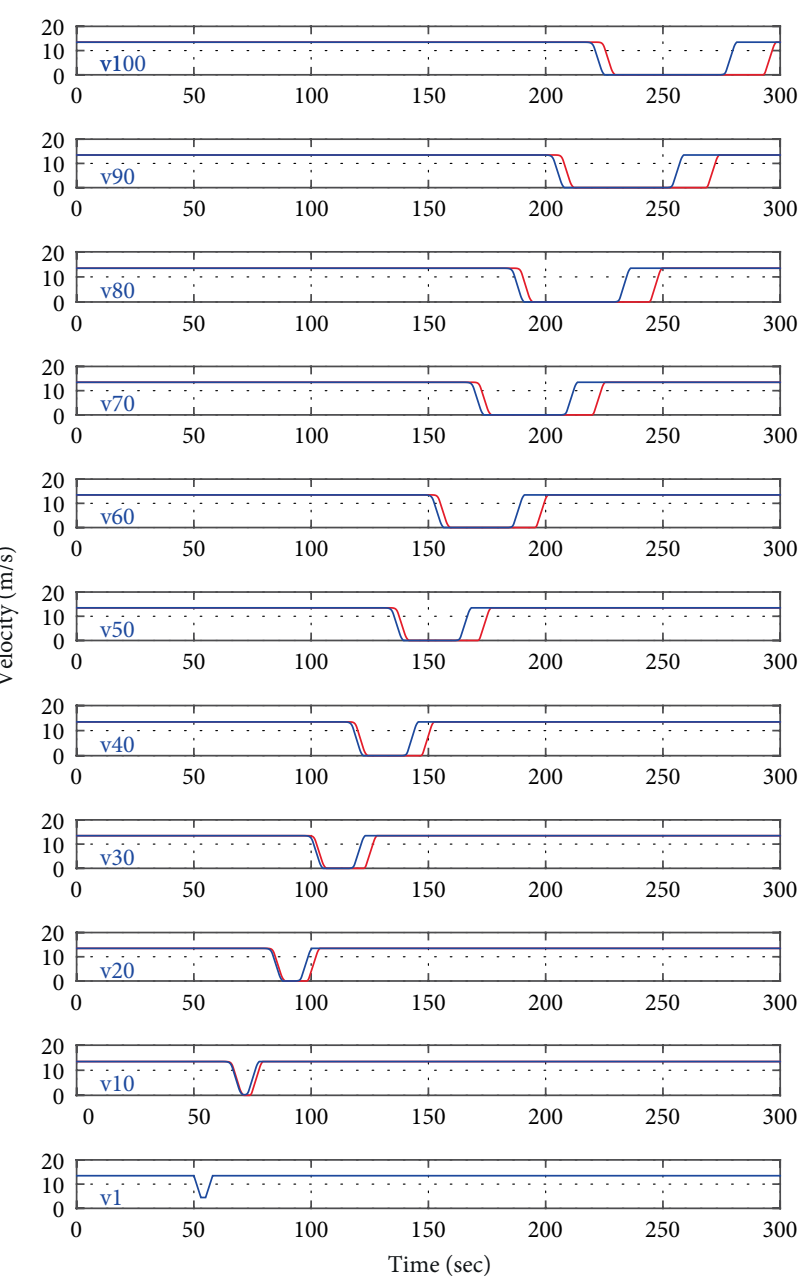

FIgURE 7: Velocity trajectories of 11 samples of vehicles in two simulations (blue lines and red lines represent simulation with only CAVs and simulation with only RVs, respectively).

fluctuation, the velocity of CAV is larger than that of the $2^{\text {nd }}$ RV. The minimum velocity of the $2^{\text {nd }} \mathrm{CAV}$ is $3.1 \mathrm{~m} / \mathrm{s}$ while that of the $2^{\text {nd }} \mathrm{RV}$ is $2.8 \mathrm{~m} / \mathrm{s}$ in the simulation. The velocity of the $2^{\text {nd }} \mathrm{CAV}$ and $2^{\text {nd }} \mathrm{RV}$ in two simulations gradually reach the maximum velocity $(13.47 \mathrm{~m} / \mathrm{s})$ at $t=60.7 \mathrm{~s}$ and $60.8 \mathrm{~s}$, respectively. This phenomenon can be explained as following.

According to formula (5), the AOV model complies with a principle of "slow-in" and "fast-out" and it means that when the preceding vehicle decelerates, deceleration of the following vehicle with AOV model is bigger than the deceleration of the following vehicle with OV model, so the following vehicle with AOV model move forward slower than the following vehicle with OV model (this is what the "slow-in"means). On the contrary, when the preceding vehicle accelerates, the following vehicle with AOV model move forward quicker than the following vehicle with OV model (this is what the "fast-out" means). Because of the difference between AOV model and OV model, difference occurs in velocity trajectories of the 2 nd vehicle in two simulations.

Velocity trajectories of 11 vehicles $\left(1^{\text {st }}, 10^{\text {th }}, 20^{\text {th }} \ldots 100^{\text {th }}\right.$ vehicles) in two simulations are shown in Figure 7. It can be 


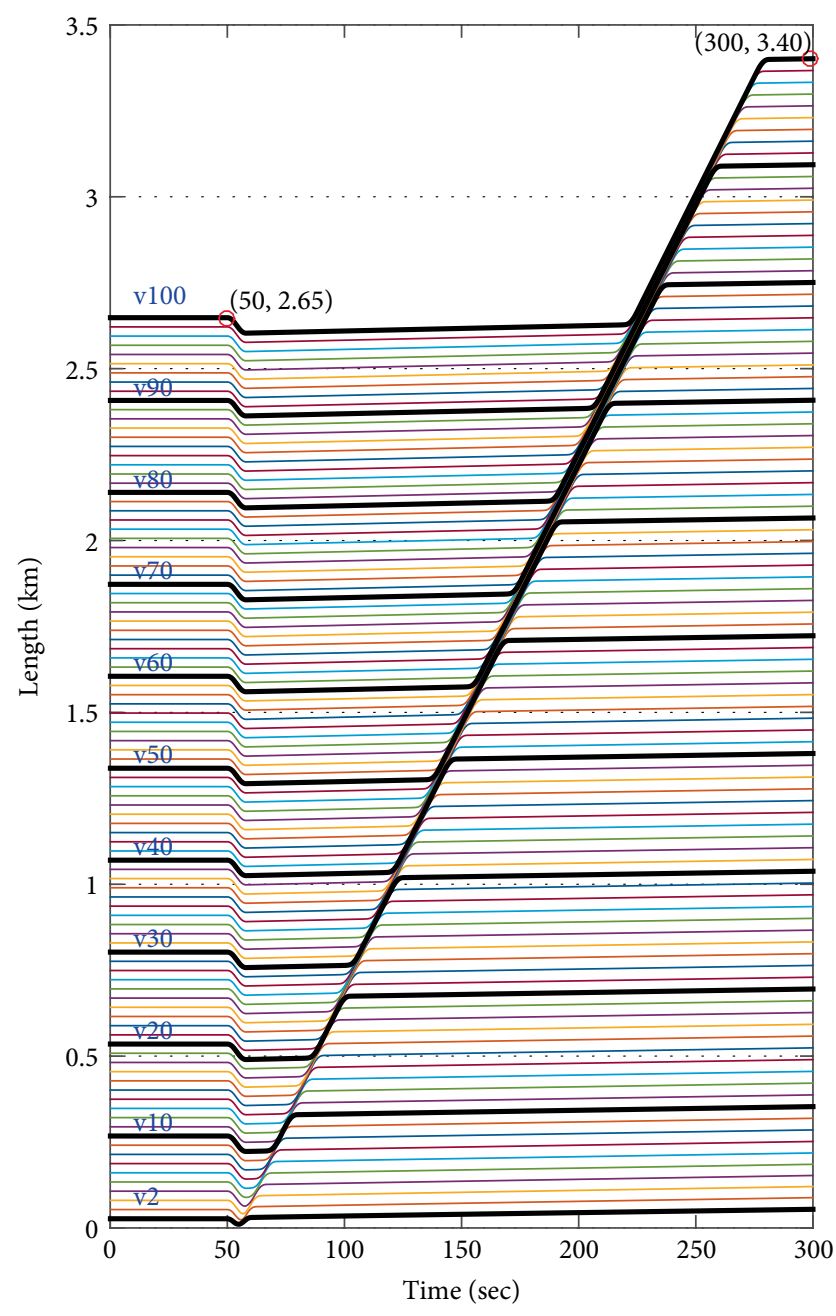

FIGURE 8: Distance between 1st vehicle and the 99 following vehicles.

seen obviously, velocity of vehicles has smaller fluctuation amplitude in simulation with only CAVs than that with same ID in simulation with only RVs when the traffic oscillations are over. Vehicles in simulation with only CAVs decelerate or stop earlier as well as reach the maximum velocity earlier than vehicles with same ID in simulation with only RVs. A simple conclusion can be obtained obviously that the CAV moving according to AOV model is helpful for mitigating traffic oscillations, and reducing velocity fluctuation of vehicle.

Figure 8 shows the platoon length between the $1^{\text {st }}$ vehicle and the 99 following vehicles. The platoon length between last vehicle $\left(100^{\text {th }}\right.$ vehicle $)$ and first vehicle $\left(1^{\text {st }}\right.$ vehicle $)$ at $t=300 \mathrm{~s}$ in the platoon is $3.40 \mathrm{~km}$ in simulation with only CAVs, while it is $3.57 \mathrm{~km}$ in simulation with only RVs, which means the length of road occupied in simulation with only CAVs is shorter than that in simulation with only RVs by $0.17 \mathrm{~km}$.

Figure 9 shows the stopping time of every vehicle and total stopping time of all vehicles in two simulations. It can be obviously seen that vehicles in simulation with only CAVs has a shorter stopping time than the vehicle with same ID in simulation with only RVs. Taking $100^{\text {th }}$ vehicle as an example, the stopping time of $100^{\text {th }}$ vehicle is $63.0 \mathrm{~s}$ in simulation with only RVs. While it is $49.6 \mathrm{~s}$ in simulation with only CAVs. In

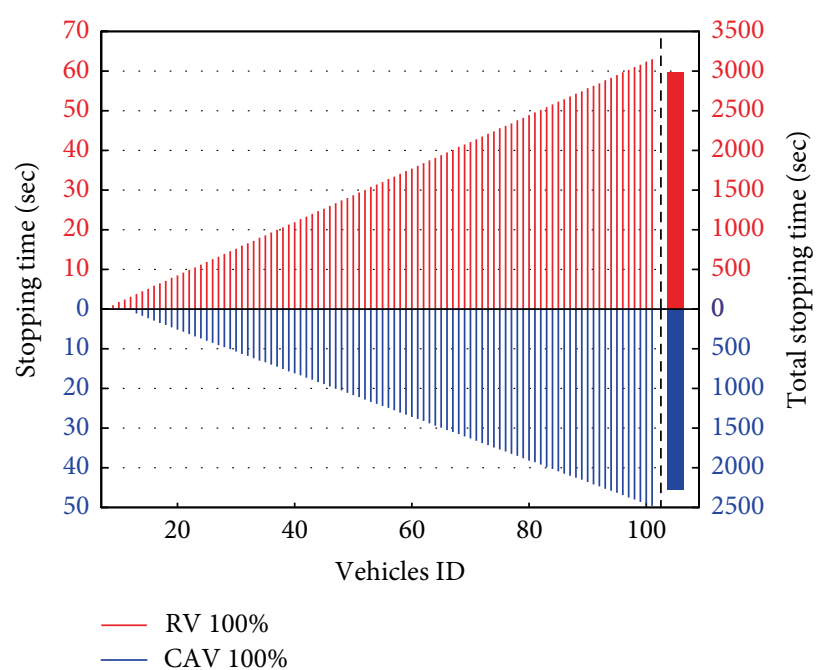

FIgURE 9: Vehicles' stopping time in two simulations.

addition, the $12^{\text {th }}$ vehicle is the first stopping vehicle in simulation with only CAVs, while the $8^{\text {th }}$ is the first stopping vehicle in simulation with only RVs. It is shown that the traffic oscillations have a larger effect on platoon in simulation with only RVs, and CAVs are benefit for the traffic oscillations mitigation in vehicle platoon. The total stopping time (227.0 s) of all vehicles in simulation with only CAVs significantly smaller than that $(298.2 \mathrm{~s})$ in simulation with only CAVs, and there is a $23.9 \%$ reduction of total stopping time of all vehicles.

Figure 10 shows the variance of velocity of vehicles in two simulations. Figure 10(a) shows the time-varying variance of velocity of all vehicles in two simulations, and it can be seen from Figure 10(a) that in the $t=0-50 \mathrm{~s}$, variance of velocity of all vehicles is 0 in all the time in two simulations. After a perturbation occurs at $t=51 \mathrm{~s}$, variance of velocity of all vehicles increases for a period and then decreases to 0 gradually in two simulations. But time-varying variance of velocity of all vehicles of simulation with only CAVs is smaller than that of simulation with only RVs in $t=51 \mathrm{~s}$ to $t=298 \mathrm{~s}$. It means the traffic flow in simulation with only CAVs has a smaller fluctuation than simulation with only RVs. As for simulation with only RVs, variance of velocity of all vehicles reaches the maximum 36.2 at $t=227.9 \mathrm{~s}$. While for simulation with only CAVs, the maximum of variance of velocity of all vehicles is 33.1 (at $t=224.2 \mathrm{~s})$.

Figure 10(b) shows the variance of velocity of every vehicle in two simulations. It can be seen that the variance of velocity of every vehicle increases with the increase of vehicle ID, it means that fluctuation of vehicles with bigger ID is bigger. The variance of velocity of every vehicle in simulation with only CAVs is smaller than the vehicles with same ID in simulation with only RVs. It means that traffic flow in simulation with only CAVs has a smaller fluctuation than simulation with only RVs.

\subsection{Numerical Simulations of Heterogeneous Traffic Flow} Containing CAVs and RVs. In Section 2 and Section 4 part 1 , simulations containing only RVs (penetration rate of CAV is 0 ) and only CAVs (penetration rate of CAV is 1 ) 


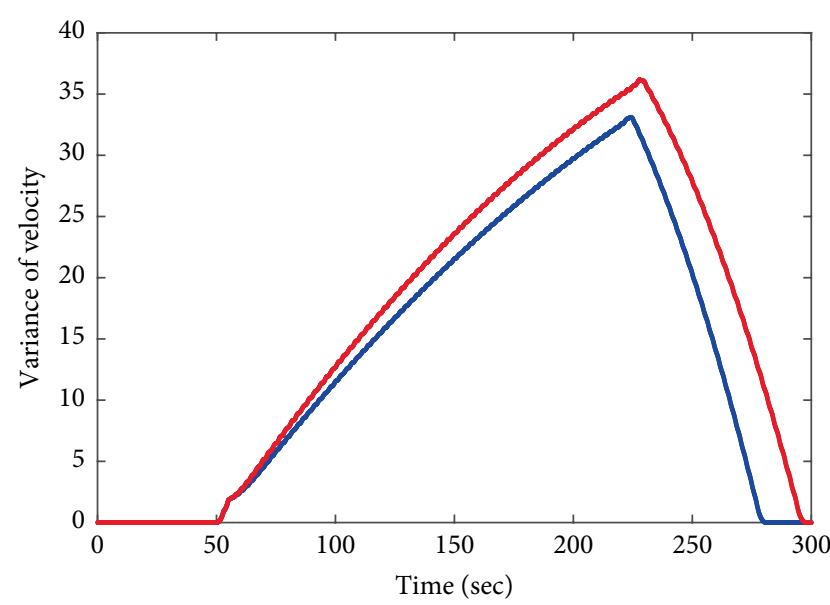

- $100 \% \mathrm{CAV}$

- $100 \% \mathrm{RV}$

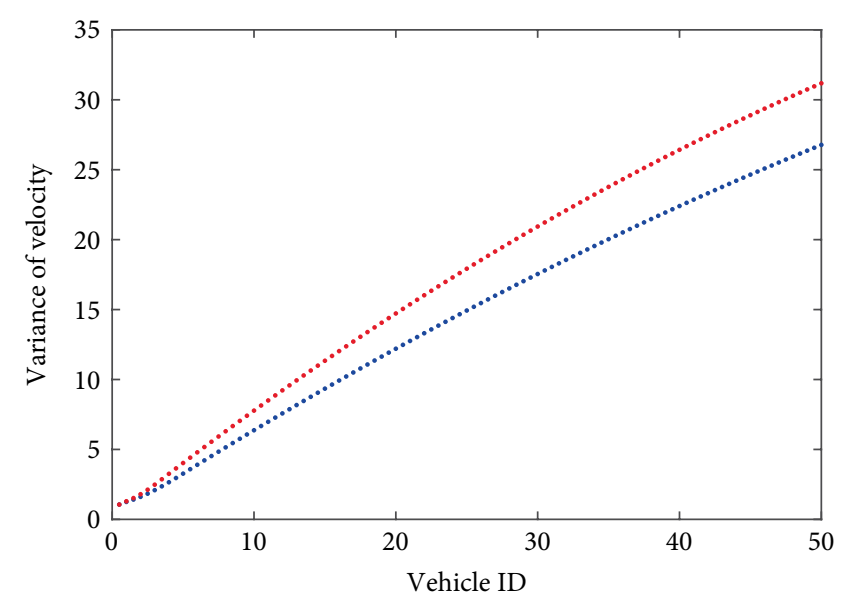

- $100 \% \mathrm{CAV}$

- $100 \% \mathrm{RV}$

(a)

(b)

FIGURE 10: Variance of velocity of vehicles. (a) Time-varying variance of velocity of all vehicles. (b) Variance of velocity of every vehicle.

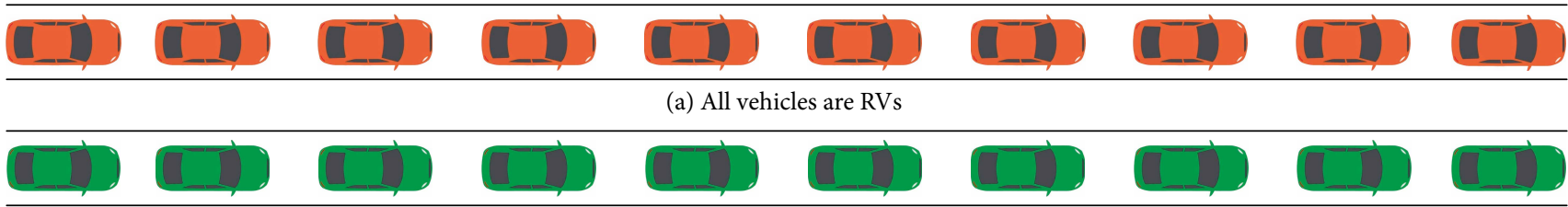

(b) All vehicles are CAVs

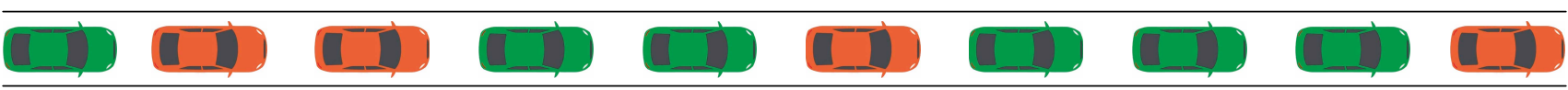

(c) Vehicles are made up of CAVs and RVs

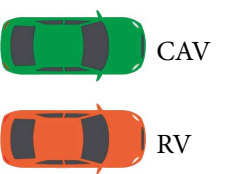

FIGURE 11: Illustrative distribution of CAVs and RVs.

are conducted, respectively, and these scenario are shown in Figures 11(a) and 11(b), respectively. In this part, we will study the heterogeneous traffic flow containing CAVs and RVs and discuss some situations that the penetration rate of CAV is bigger than 0 and smaller than 1. Figure 11(c) displays the scenario that CAVs and RVs randomly distribute and the penetration rate of CAV is 0.6 (we display only 10 vehicles in this illustrative figure, real simulations contain 100 vehicles).

When all vehicles are RVs (Figure 11(a)), all RVs move according to OV model except the leading vehicle of the platoon, the leading vehicle move according to specified velocity and acceleration. While, when all vehicles are CAVs (Figure 11(b)), all CAVs move according to AOV model except the leading vehicle of the platoon. However, when a vehicle platoon contains RVs and CAVs, how many vehicles move according to AOV model depends on the penetration rate of $\mathrm{CAV}$ and the distribution of CAVs. If the preceding vehicle is a CAV, the following CAV move according to AOV model. On the contrary, if the preceding vehicle is a $\mathrm{RV}$, the following
CAV move according to OV model. In general, the bigger the penetration rate of CAV is, the more CAV pairs or consecutive CAVs the platoon contains, and the more vehicles move according to AOV model.

In this part, the simulations are conducted with the situations that penetration rates of CAV are $0.2,0.4,0.6$, and 0.8 , and five simulations are conducted for every penetration rate, and CAVs distribute randomly in every simulation. Finally, three indexes are calculated and analysed with the average value of every five simulations of same scenario with different penetration rates, the three indexes are total stopping time of all vehicles, road length occupied by vehicles (the space between fist vehicle and the last vehicle in the platoon) and variance of velocity of all vehicles and they are shown in Figures 12(a)$12(\mathrm{c})$, respectively.

A trend can be easily seen from Figure 12(a) that the total stopping time of all vehicles decreases with the increase of penetration rate of CAV. The total stopping time is $2982 \mathrm{~s}$, 2951.4 s, 2867.6 s, 2716 s, 2487.2 s, and $2270 \mathrm{~s}$ in the simulations with penetration rates are $0,0.2,0.4,0.6,0.8$, and 1 


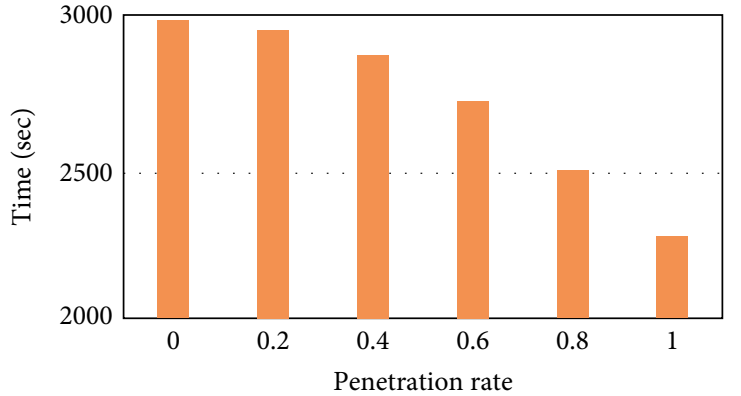

(a)

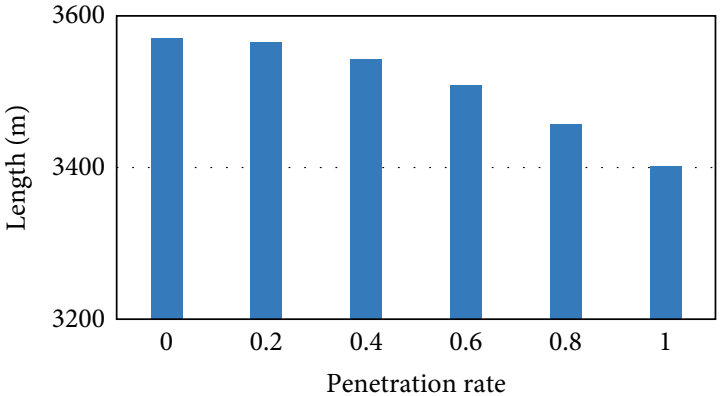

(b)

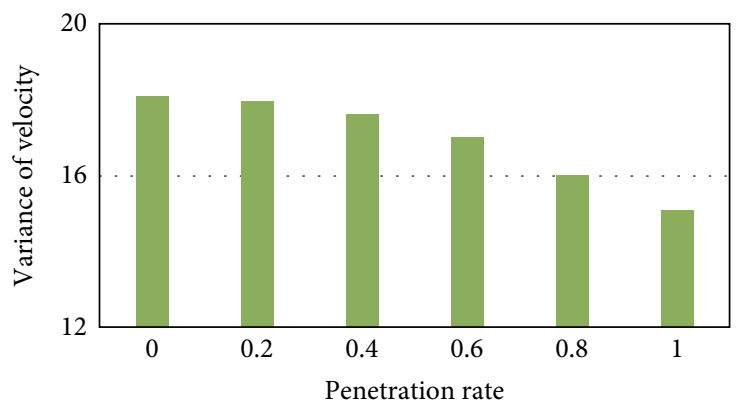

(c)

FIGURE 12: Three indexes of simulations with different penetration rate of CAV. (a) Total stopping time of all vehicles. (b) Road length occupied by vehicles. (c) Variance of velocity of all vehicles.

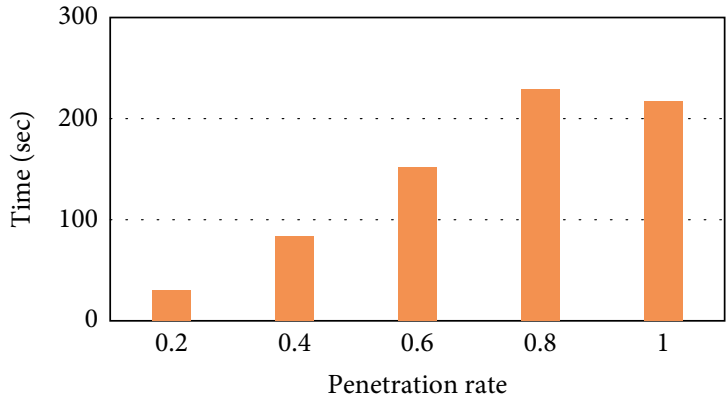

(a)

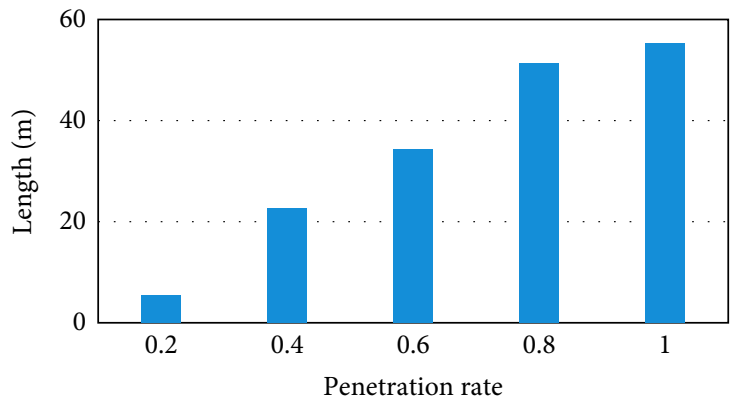

(b)

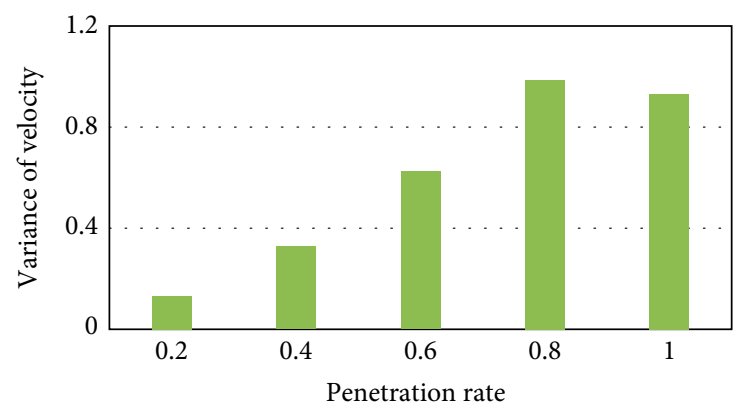

(c)

FIGURE 13: The improvement of three indexes of simulations under each penetration rate of CAV relative to its' previous penetration rate. (a) Total stopping time of all vehicles. (b) Road length occupied by vehicles. (c) Variance of velocity of all vehicles.

respectively. It can be seen from the Figures 12(b) and 12(c), other two indexes, road length occupied by vehicles and variance of velocity of all vehicles have the same trends with the index of total stopping time, and these two indexes decrease with the increase of penetration rate of CAV too. As for the index of road length occupied by vehicles, it is 18.1, 17.9, $17.6,17.0,16.0$, and 15.1 in the simulations with penetration rate are $0,0.2,0.4,0.6,0.8$, and 1 , respectively. As for the index of variance of velocity of all vehicles, it can reflect the degree of velocity fluctuation, and the small velocity 
fluctuation is better for the stability of traffic flow as well as throughput. The index of variance of velocity of all vehicles is $18.1,17.9,17.6,17.0,16.0$, and 15.1 in the simulations with penetration rates are $0,0.2,0.4,0.6,0.8$ and 1 , respectively. A previous conclusion can be obtained that these three indexes improve with the increase of penetration rate of CAV, and this means more CAVs are better for reducing the fluctuation of velocity and mitigating traffic oscillations.

Figures 13(a)-13(c) show how much improvement of each index under each penetration rate of CAV relative to its' previous penetration rate.

From Figure 13(a), we can see that the improvements of the index of total stopping time of all vehicles have big differences under each penetration rate relative to its' previous penetration rate. It can be seen that there is an obvious trend that the improvements of the index increase with the increase of penetration rate, although the improvement under penetration rate of 0.8 relative to its previous penetration rate of 0.6 is little bigger than the improvement under penetration rate of 1 relative to its' previous penetration rate of 0.8 . When the penetration rate of $\mathrm{CAV}$ increases from 0 to 0.2 , the improvement of total stopping time of all vehicles is $30.6 \mathrm{~s}$, and this improvement is smallest. While, when the penetration rate of CAV increases from 0.6 to 0.8 , the improvement of total stopping time of all vehicles is $228.8 \mathrm{~s}$, and this improvement is largest. When the penetration rate of CAV increases from 0.2 to 0.4 , 0.4 to 0.6 , and 0.8 to 1 , the improvement of total stopping time of all vehicles is $83.8 \mathrm{~s}, 151.6 \mathrm{~s}$, and $217.2 \mathrm{~s}$, respectively. The main reason is that with the increase of penetration rate of $\mathrm{CAV}$, there are more CAVs as well as CAV pairs or consecutive CAVs in the vehicle platoon. For every CAV pairs, the following CAV behind its' preceding CAV moves forward according to AOV model, and the AOV model reflects the principle of "slow-in" and "fast-out," and this principle is good for mitigating traffic oscillations. So the bigger the penetration rate is, the more the CAV pairs or consecutive CAVs will be, the smaller the traffic oscillations will be and the more the improvement of the total stopping time of all vehicles will be.

It can be seen from the Figures 13(b) and 13(c), other two indexes, road length occupied by vehicles and variance of velocity of all vehicles have the same trends with the index of total stopping time under each penetration rate of CAV relative to its' previous penetration rate, and these two indexes increase with the increase of penetration rate of CAV too. It should be noted that the improvement of road length occupied by vehicles under penetration rate of 0.8 relative to its' previous penetration rate 0.6 is little smaller than the improvement of road length occupied by vehicles under penetration rate of 1 relative to its' previous penetration rate 0.8 . When the penetration rate of CAV increases from 0 to $0.2,0.2$ to $0.4,0.4$ to $0.6,0.6$ to 0.8 , and 0.8 to 1 , the improvement of road length occupied by vehicles is $5.4 \mathrm{~m}, 22.6 \mathrm{~m}, 34.4 \mathrm{~m}, 51.4 \mathrm{~m}$, and $55.2 \mathrm{~m}$, respectively, and the improvement of variance of velocity of all vehicles is $0.1,0.3,0.6,1.0$, and 0.9 , respectively.

\section{Conclusions}

This paper aim at designing control strategy for CAVs to mitigate traffic oscillations and improve traffic efficiency and studying the effectiveness of the control strategy and analysing the characteristics of traffic flow made up of only CAVs or made up of CAVs and RVs when traffic oscillations appear in traffic flow. Considering that the CAVs can share real-time state information with each other and the idea of "slow-in" and "fast-out" is benefit for mitigating traffic oscillations, acceleration of preceding CAV is introduced into OV model to control the longitudinal motion of CAV. Numerical simulations were conducted to simulate the dynamic of traffic flow when traffic oscillations propagate in vehicle platoon. The simulations consider the situations of homogeneous traffic flow containing only CAVs and heterogeneous traffic flow containing CAVs and RVs, and different penetration rates of $\mathrm{CAV}$ are considered in the simulations of homogeneous traffic flow. Simulation results are analysed from the aspects of vehicles' velocity fluctuations, stopping time, and length of road occupied. Compared with the situation that the vehicle platoon containing only RVs, vehicles have smaller velocity fluctuations less stopping time and length of road occupied is shorter when the vehicle platoon contains CAVs. With the increase of penetration rates of $\mathrm{CAV}$, the improvement of the traffic flow is more obvious. An obvious conclusion is that CAVs in a vehicle platoon is helpful to mitigate traffic oscillations and improving traffic flow, and the control strategy of CAV in this paper is effective.

Due to the difficulty of testing the performance of CAV technologies in field under our current situation, this study is conducted from the level of simulation. In the future, we could use multiple driving simulators to study the effectiveness of control strategy for CAVs and analyse traffic oscillations in heterogeneous traffic flow containing CAVs and RVs.

\section{Data Availability}

If anyone needs the data in this paper, you can contact E-mail 17114223@bjtu.edu.cn.

\section{Conflicts of Interest}

The authors declare that there is no conflicts of interest regarding the publication of this paper.

\section{Acknowledgments}

This work was supported by the fundamental research funds for the Central Universities [grant number 2019JBM041] and the National Key R\&D Program of China [grant number 2018YFB1600703]. 


\section{References}

[1] Y. Sugiyama, M. Fukui, M. Kikuchi et al., "Traffic jams without bottlenecks-experimental evidence for the physical mechanism of the formation of a jam," New Journal of Physics, vol. 10, pp. 1-7, 2008.

[2] J. A. Laval and L. Leclercq, "A mechanism to describe the formation and propagation of stop-and-go waves in congested freeway traffic," Philosophical Transactions Mathematical Physical \& Engineering Sciences, vol. 368, no. 1928, pp. 45194541, 2010.

[3] J. A. Laval, C. S. Toth, and Y. Zhou, "A parsimonious model for the formation of oscillations in car-following models," Transportation Research Part B: Methodological, vol. 70, pp. 228-238, 2014.

[4] D. J. Chen, S. Ahn, J. Laval, and Z. D. Zheng, "On the periodicity of traffic oscillations and capacity drop: the role of driver characteristics," Transportation Research Part B: Methodological, vol. 59, pp. 117-136, 2014.

[5] D. J. Chen, J. Laval, Z. D. Zheng, and S. Ahn, "A behavioral car-following model that captures traffic oscillations," Transportation Research Part B: Methodological, vol. 46, no. 6, pp. 744-761, 2012.

[6] US Federal Highway Administration, "Next Generation Simulation Program (NGSIM)," https://ops.fhwa.dot.gov/ trafficanalysistools/ngsim.htm, December 2006.

[7] R. Nishi, A. Tomoeda, K. Shimura, and K. Nishinarit, "Theory of jam-absorption driving," Transportation Research Part B: Methodological, vol. 50, pp. 116-129, 2013.

[8] Z. B. He, L. Zheng, L. Y. Song, and N. Zhu, "A jam-absorption driving strategy for mitigating traffic oscillations," IEEE Transactions on Intelligent Transportation Systems, vol. 18, no. 4, pp. 802-813, 2017.

[9] Y. Taniguchi, R. Nishi, T. Ezaki, and K. Nishinari, "Jamabsorption driving with a car-following model," Physica A, vol. 433, pp. 304-315, 2015.

[10] C. F. Caruntu, C. Copot, C. Lazar, and R. D. Keyser, "Longitudinal control of vehicle platoons for stop-and-go waves mitigation," in 18th International Conference on System Theory Control and Computing, pp. 664-669, Sinaia, Romania, October 2014.

[11] J. Lioris, R. Pedarsani, F. Y. Tascikaraoglu, and P. Varaiya, "Platoons of connected vehicles can double throughput in urban roads," Transportation Research Part C: Emerging Technologies, vol. 77, no. 15, pp. 292-305, 2017.

[12] B. Khondaker and L. Kattan, "Variable speed limit: a microscopic analysis in a connected vehicle environment," Transportation Research Part C: Emerging Technologies, vol. 58, pp. 146-159, 2015.

[13] S. I. Guler, M. Menendez, and L. Meier, "Using connected vehicle technology to improve the efficiency of intersections," Transportation Research Part C: Emerging Technologies, vol. 46, pp. 121-131, 2014.

[14] H. Farah and H. N. Koutsopoulos, "Do cooperative systems make drivers' car-following behaviour safer?" Transportation Research Part C: Emerging Technologies, vol. 41, pp. 61-72, 2014.

[15] A. J. Khattak and B. Wali, "Analysis of volatility in driving regimes extracted from basic safety messages transmitted between connected vehicles," Transportation Research Part C: Emerging Technologies, vol. 84, pp. 48-73, 2017.
[16] T. Q. Tang, W. F. Shi, H. Y. Shang, and Y. P. Wang, "A new car-following model with consideration of inter-vehicle communication," Nonlinear Dynamics, vol. 76, no. 4, pp. 2017 2023, 2014.

[17] T. Q. Tang, W. F. Shi, H. Y. Shang, and Y. P. Wang, "An extended car-following model with consideration of the reliability of inter-vehicle communication," Measurement, vol. 58, pp. 286293, 2014.

[18] S. W. Yu and Z. K. Shi, "Dynamics of connected cruise control systems considering velocity changes with memory feedback," Measurement, vol. 64, pp. 34-48, 2015.

[19] J. Zhao and P. Li, "An extended car-following model with consideration of speed guidance at intersections," Physica A, vol. 461, pp. 1-8, 2016.

[20] D. Jia and D. Ngoduy, "Enhanced cooperative car-following traffic model with the combination of V2V and V2I communication," Transportation Research Part B: Methodological, vol. 90, pp. 172-191, 2016.

[21] M. A. S. Kamal, J. I. Imura, T. Hayakawa, A. Ohata, and K. Aihara, "Smart driving of a vehicle using model predictive control for improving traffic flow," IEEE Transactions on Intelligent Transportation Systems, vol. 15, no. 2, pp. 878-888, 2014.

[22] M. Wang, W. Daamen, S. P. Hoogendoorn, and B. V. Arem, "Connected variable speed limits control and car-following control with vehicle-infrastructure communication to resolve stop-and-go waves," Journal of Intelligent Transportation Systems, vol. 20, no. 6, pp. 559-572, 2016.

[23] R. E. Stern, S. Cui, M. L. D. Monache et al., "Dissipation of stop-and-go waves via control of autonomous vehicles: field experiments," Transportation Research Part C: Emerging Technologies, vol. 89, pp. 205-221, 2017.

[24] M. Wang, W. Daamen, S. P. Hoogendoorn, and B. V. Arem, "Cooperative car-following control: Distributed algorithm and impact on moving jam features," IEEE Transactions on Intelligent Transportation Systems, vol. 17, no. 5, pp. 1459-1471, 2016.

[25] J. I. Ge, S. S. Avedisov, C. R. He, W. B. Qin, M. Sadeghpour, and G. Orosz, "Experimental validation of connected automated vehicle design among human-driven vehicles," Transportation Research Part C: Emerging Technologies, vol. 91, pp. 335-352, 2018.

[26] R. E. Chandler, R. Herman, and E. W. Montroll, "Traffic dynamics: studies in car following," Operations Research, vol. 6, no. 2, pp. 165-184, 1958.

[27] D. C. Gazis, R. Herman, and R. W. Rothery, "Nonlinear followthe-leader models of traffic flow," Operations Research, vol. 9, no. 4, pp. 545-567, 1961.

[28] G. F. Newell, "Nonlinear effects in the dynamics of car following," Operations Research, vol. 9, no. 2, pp. 209-229, 1961.

[29] M. Bando, K. Hasebe, A. Nakayama, and Y. Sugiyarna, "Dynamical model of traffic congestion and numerical simulation," Physical Review E, vol. 51, no. 2, pp. 1035-1042, 1995.

[30] D. Helbing and B. Tilch, "Generalized force model of traffic dynamics," Physical Review E, vol. 58, no. 1, pp. 133-138, 1998.

[31] R. Jiang, Q. S. Wu, and Z. J. Zhu, "Full velocity difference model for a car-following theory," Physical Review E, vol. 64, no. 1, pp. 7101-7104, 2001. 
[32] G. H. Peng and D. H. Sun, "A dynamical model of car-following with the consideration of the multiple information of preceding cars," Physics Letters A, vol. 374, no. 15-16, pp. 1694-1698, 2010.

[33] T. Q. Tang, C. Y. Li, and H. J. Huang, "A new car-following model with the consideration of the driver's forecast effect," Physics Letters A, vol. 374, no. 38, pp. 3951-3956, 2010.

[34] T. Q. Tang, J. G. Li, H. J. Huang, and X. B. Yang, "A car-following model with real-time road conditions and numerical tests," Measurement, vol. 48, pp. 63-76, 2014.

[35] H. X. Ge, X. P. Meng, H. B. Zhu, and Z. P. Li, "Feedback control for car following model based on two-lane traffic flow," Physica A, vol. 408, pp. 28-39, 2014.

[36] W. X. Zhu and L. D. Zhang, "A speed feedback control strategy for car-following model," Physica A, vol. 413, pp. 343-351, 2014. 


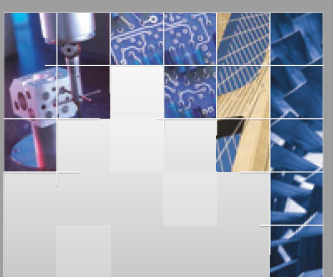

\section{Enfincering}
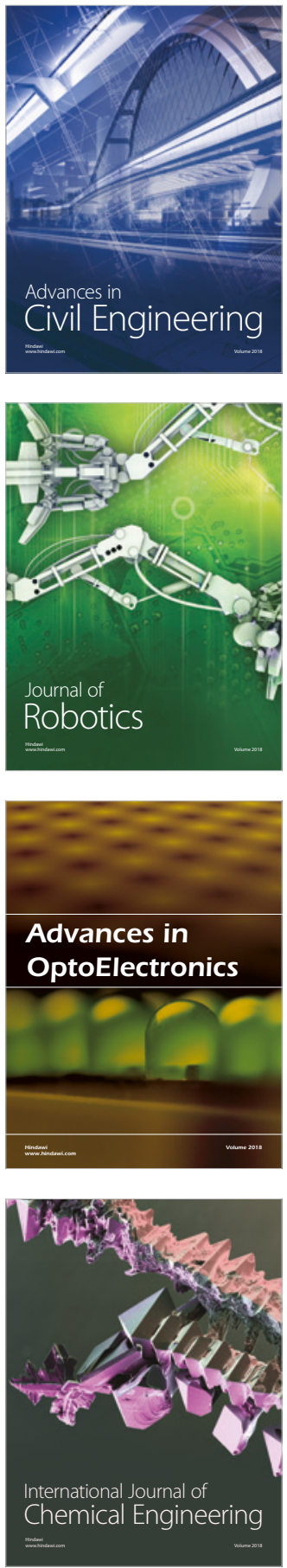

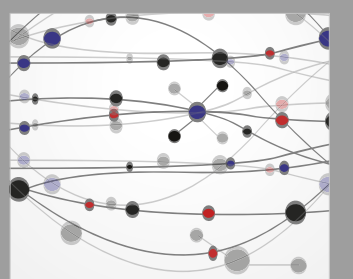

\section{Rotating \\ Machinery}

The Scientific World Journal

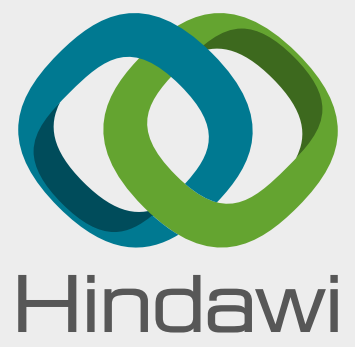

Submit your manuscripts at

www.hindawi.com
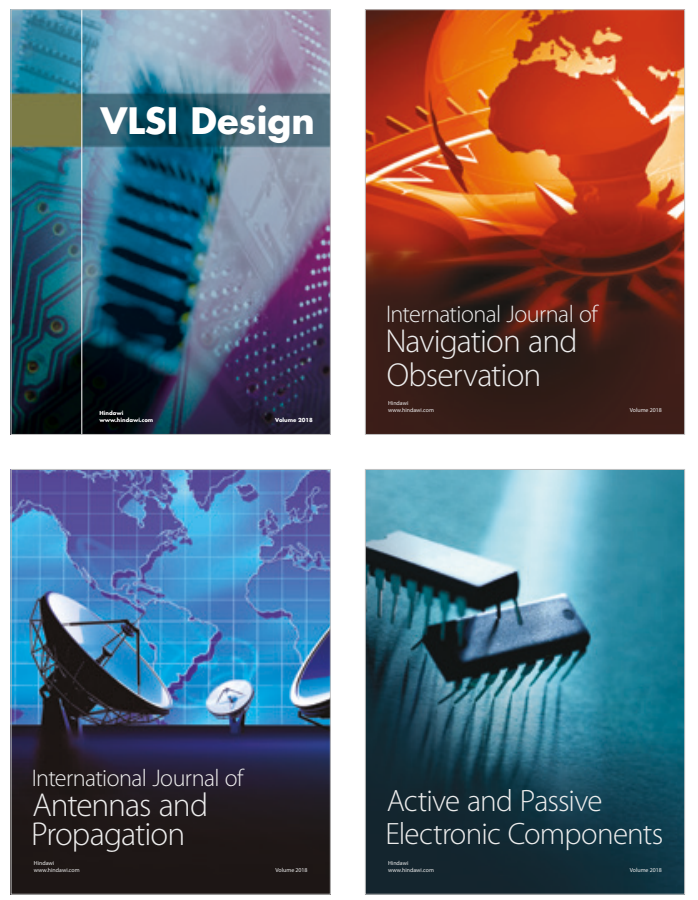
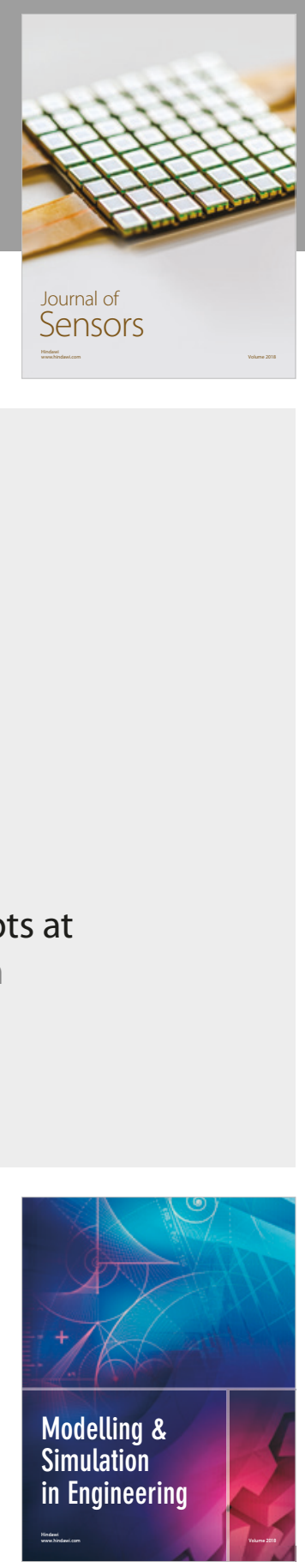

\section{Advances \\ Multimedia}
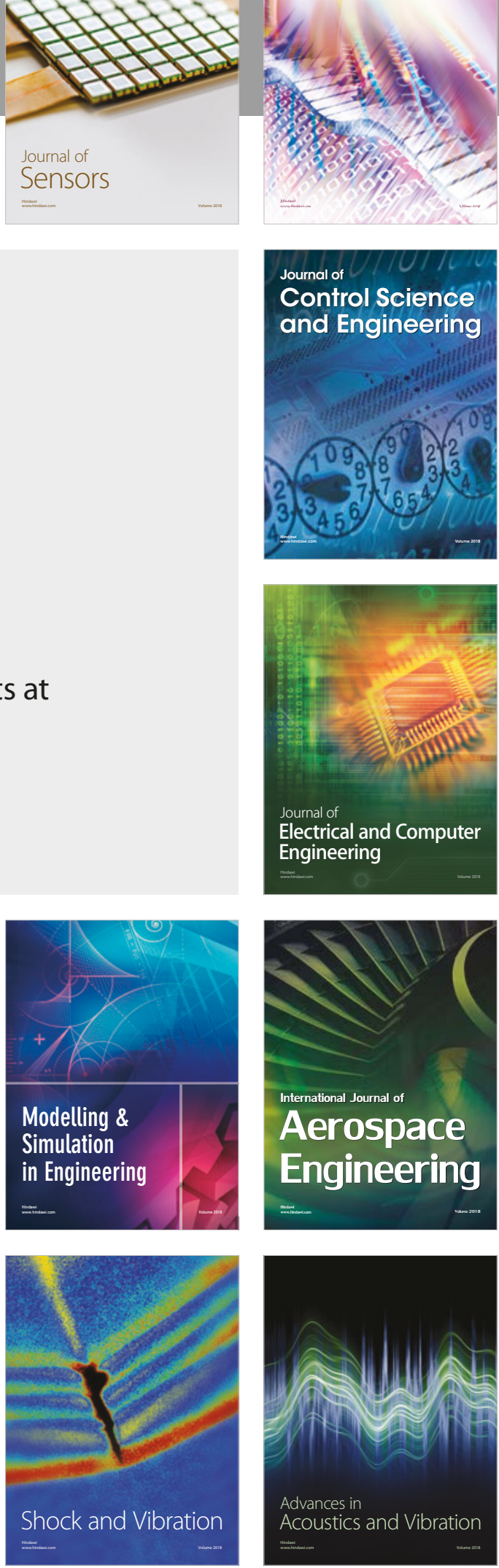